\title{
ON PLANARITY OF GRAPHS ON WEYL GROUPS
}

\author{
S. A. YOUSSEF AND S. G. HULSURKAR
}

\begin{abstract}
A graph is constructed whose vertices are elements of a Weyl group and the edges are defined through nonvanishing of Weyl's dimension polynomial at the point associated with two elements of the Weyl group. We study the planarity of such graphs on Weyl groups whose associated root system is irreducible. These graphs include four families of infinite number of graphs. We show that very few graphs, essentially five of them, are planar.
\end{abstract}

\section{Introduction and Notation}

We construct a graph on Weyl groups and determine the planarity of these graphs. The definition of an edge of the graph involves the underlying root system of the Weyl group. The origin of such a definition can be seen in [3]. A new partial order on Weyl groups was introduced there to prove the Verma's conjecture on Weyl's dimension polynomial. This partial order is used to define the graph structure on a Weyl group. The matrix studied in [1] is nothing but the incidence matrix of our graphs here. The study of such graphs has been done in [5]. We give here the results on the planarity of graphs on Weyl groups whose associated root system is irreducible. We describe briefly the root system and its Weyl group and refer to [4] for details.

Let $\Phi$ be a root system in a Euclidean space $E$ of dimension $n$ with positive definite inner product (,). For $\alpha \in \Phi$, let $R_{\alpha}$ be the reflection given by $\lambda R_{\alpha}=\lambda-\left(\lambda, \alpha^{V}\right) \alpha$, for $\lambda \in E$ and where $\alpha^{V}=2 \alpha /(\alpha, \alpha)$. Let $\alpha_{1}, \alpha_{2}, \ldots, \alpha_{n}$ be simple roots of $\Phi$. Then $W$ is generated by $R_{i}=R_{\alpha_{i}}, i=1,2, \ldots, n$. Any element of $W$ can be written as a product of finite number of the generators $R_{i}$. Let $\ell(\sigma)$ be the length of $\sigma$ defined as the minimum number of generators $R_{i}$ required to express $\sigma$ as a product of the generators. Suppose $\lambda_{1}, \lambda_{2}, \ldots, \lambda_{n}$ are fundamental weights of $\Phi$, i.e., they are defined by $\left(\lambda_{i}, \alpha_{j}^{V}\right)=\delta_{i j}$ (Kronecker delta). For $\sigma \in W$, define $I_{\sigma}=\left\{i \mid 1 \leq i \leq n, \ell\left(\sigma R_{i}\right)<\ell(\sigma)\right\}$ and $\delta_{\sigma}=\sum_{i \in I_{\sigma}} \lambda_{i}$. The weight vector $\epsilon_{\sigma}$ associated with each $\sigma \in W$ is defined by

Received May 13, 1994.

1991 Mathematics Subject Classification. 05C25

Key words and phrases. Weyl groups, root system, planarity of a graph. 
$\epsilon_{\sigma}=\delta_{\sigma} \sigma^{-1}$. Let $D(\lambda)$ for $\lambda \in E$ be the Weyl's dimension polynomial. Then

$$
D(\lambda)=\frac{\prod_{\alpha \in \Phi^{+}}\left(\lambda, \alpha^{V}\right)}{\prod_{\alpha \in \Phi^{+}}\left(\delta, \alpha^{V}\right)}
$$

for $\lambda \in E$ and where $\delta=\sum_{i=1}^{n} \lambda_{i}$ and $\Phi^{+}$is the set of positive roots.

\section{A Graph on W}

We define a graph $\Gamma(W(\Phi))$ whose vertices are elements of $W$, the Weyl group of the root system $\Phi$, and the edges are defined by the root system $\Phi$. We also write $\Gamma(W)$ or $\Gamma(\Phi)$ for $\Gamma(W(\Phi))$. A point $\lambda \in E$ is called $W$-regular if $D(\lambda) \neq 0$ or that is same as $\lambda$ is in the interior of a Weyl chamber relative to $\Phi$. Let $\sigma_{0}$ be the unique element of maximal length in $W$. For distinct $\sigma, \tau \in W$ the unordered pair $(\sigma, \tau)$ is an edge in $\Gamma(\Phi)$ iff either $-\epsilon_{\sigma \sigma_{0}}+\epsilon_{\tau}$ or $-\epsilon_{\tau \sigma_{0}}+\epsilon_{\sigma}$ is $W$-regular. It is shown in [3] that both $-\epsilon_{\sigma \sigma_{0}}+\epsilon_{\tau}$ and $-\epsilon_{\tau \sigma_{0}}+\epsilon_{\sigma}$ cannot be regular. Thus we have a graph as defined in [2]. In general, if $-\epsilon_{\sigma \sigma_{0}}+\epsilon_{\tau}$ is $W$-regular then we write $\sigma \rightarrow \tau$ in $W$. It is easy to see that $\sigma \rightarrow \sigma$ in $W$ for all $\sigma \in W$, after noting, that $-\epsilon_{\sigma \sigma_{0}}=\left(\delta-\delta_{\sigma}\right) \sigma^{-1}[2]$. Further, $(\sigma, \tau)$ is an edge in $\Gamma(\Phi)$ iff $\sigma \neq \tau$ and either $\sigma \rightarrow \tau$ or $\tau \rightarrow \sigma$ in $W$. In fact, the relation $\sigma \rightarrow \tau$ in $W$ gives a directed graph on $W$, which is investigated elsewhere.

Let $J$ be a subset of $I=\{1,2, \ldots, n\}$. The subgroup $W_{J}$ generated by $R_{j}, j \in J$ is again a Weyl group, corresponding to the root system $\Phi_{J}$ whose simple roots are $\alpha_{j}$, $j \in J$ in the subspace $E_{J}$ of $E$. The root system $\Phi_{J}$ need to be irreducible. Note that $W=W_{I}$.

Lemma. Let $J$ be a nonempty subset of $I$ and $W_{J}$ be the corresponding Weyl group. Let $\sigma, \tau \in W_{J}$. Then $(\sigma, \tau)$ is an edge in $\Gamma\left(W_{J}\right)$ iff $(\sigma, \tau)$ is an edge in $\Gamma(W)$.

Proof. For any nonempty subset $J$ of $I$, let $\delta_{J}=\sum_{j \in J} \lambda_{j}$ and $\sigma_{J}$ be the unique element of maximal length in $W_{J}$. Then $\sigma_{I}$ is same as $\sigma_{0}$ and $\delta_{I}=\delta$. It has been shown [3] that for $\sigma \in W,-\epsilon_{\sigma \sigma_{0}}=\left(\delta-\delta_{\sigma}\right) \sigma^{-1}$. Then for $\sigma \in W_{J},-\epsilon_{\sigma \sigma_{J}}=\left(\delta_{J}-\delta_{\sigma}\right) \sigma^{-1}$. Every point in $E$ has a unique image in the closure of the fundamental Weyl chamber. Suppose $\bar{\delta}_{J}$ is the image of $-\epsilon_{\sigma \sigma_{J}}+\epsilon_{\tau}$ in the fundamental Weyl chamber for $W_{J}$, then

$$
\bar{\delta}_{J}=\sum_{j \in J} n_{j} \lambda_{j}
$$

where $n_{j}$ are nonnegative integers. Therefore, $-\epsilon_{\sigma \sigma_{J}}+\epsilon_{\tau}=\left(\delta_{J}-\delta_{\sigma}\right) \sigma^{-1}+\delta_{\tau} \tau^{-1}=\bar{\delta}_{J} \rho^{-1}$, for some $\rho \in W_{J}$. We recall that, for $\sigma, \tau \in W_{J},-\epsilon_{\sigma \sigma_{J}}+\epsilon_{\tau}$ is $W_{J}$-regular iff $-\epsilon_{\sigma \sigma_{J}}+\epsilon_{\tau}$ is in the interior of a Weyl chamber for the root system $\Phi_{J}$. We conclude that $-\epsilon_{\sigma \sigma_{J}}+\epsilon_{\tau}$ is $W_{J}$-regular iff $n_{j}>0$ for $j \in J$ in Eq.(1). Now $-\epsilon_{\sigma \sigma_{J}}+\epsilon_{\tau}=\left(\delta_{I}-\delta_{\sigma}\right) \sigma^{-1}+\delta_{\tau} \tau^{-1}=\left(\delta_{J}+\right.$ $\left.\delta_{I-J}-\delta_{\sigma}\right) \sigma^{-1}+\delta_{\tau} \tau^{-1}=\left(\delta_{J}-\delta_{\sigma}\right) \sigma^{-1}+\delta_{I-J} \sigma^{-1}+\delta_{\tau} \tau^{-1}=\bar{\delta}_{J} \rho^{-1}+\delta_{I-J}=\left(\bar{\delta}_{J}+\delta_{I-J}\right) \rho^{-1}$, 
since $\sigma, \tau \in W_{J}$ and $\lambda_{i} R_{i}=\lambda_{i}$ for $j \neq i$. Whence $-\epsilon_{\sigma \sigma_{I}}+\epsilon_{\tau}$ is $W$-regular iff $n_{j}>0$ for $j \in J$ in Eq. (1) iff $-\epsilon_{\sigma \sigma_{I}}+\epsilon_{\tau}$ is $W_{J}$-regular.

Corollary. $\Gamma\left(W_{J}\right)$ is induced subgraph of $\Gamma(W)$.

\section{Trreducible Root System}

Here we consider only irreducible root system since an arbitrary root system $\Phi$ is the union of irreducible root systems and the Weyl group $W$ of $\Phi$ is the direct product of the Weyl groups of the irreducible root systems. If $\Phi$ is an irreducible root system then it is uniquely determined by the Dynkin diagram given in Fig.1. The Dynkin diagram also determines the Weyl group associated with the root system. If the root system $\Phi$ is of type $A_{n}(n \geq 1)$, we write the graph $\Gamma(W(\Phi))$ as $\Gamma\left(A_{n}\right)$ for $n \geq 1$. Similarly, $\Gamma\left(B_{n}\right)$ for $n \geq 2, \Gamma\left(C_{n}\right)$ for $n \geq 3, \Gamma\left(D_{n}\right)$ for $n \geq 4, \Gamma\left(E_{6}\right), \Gamma\left(E_{7}\right), \Gamma\left(E_{8}\right), \Gamma\left(F_{4}\right)$, and $\Gamma\left(G_{2}\right)$ denote the graph on Weyl group associated with the respective root system. It should be noted that the Weyl groups of the root systems of type $B_{n}$ and $C_{n}$ for $n \geq 3$ are same but $\Gamma\left(B_{n}\right)$ and $\Gamma\left(C_{n}\right)$ are different as the root systems are different.
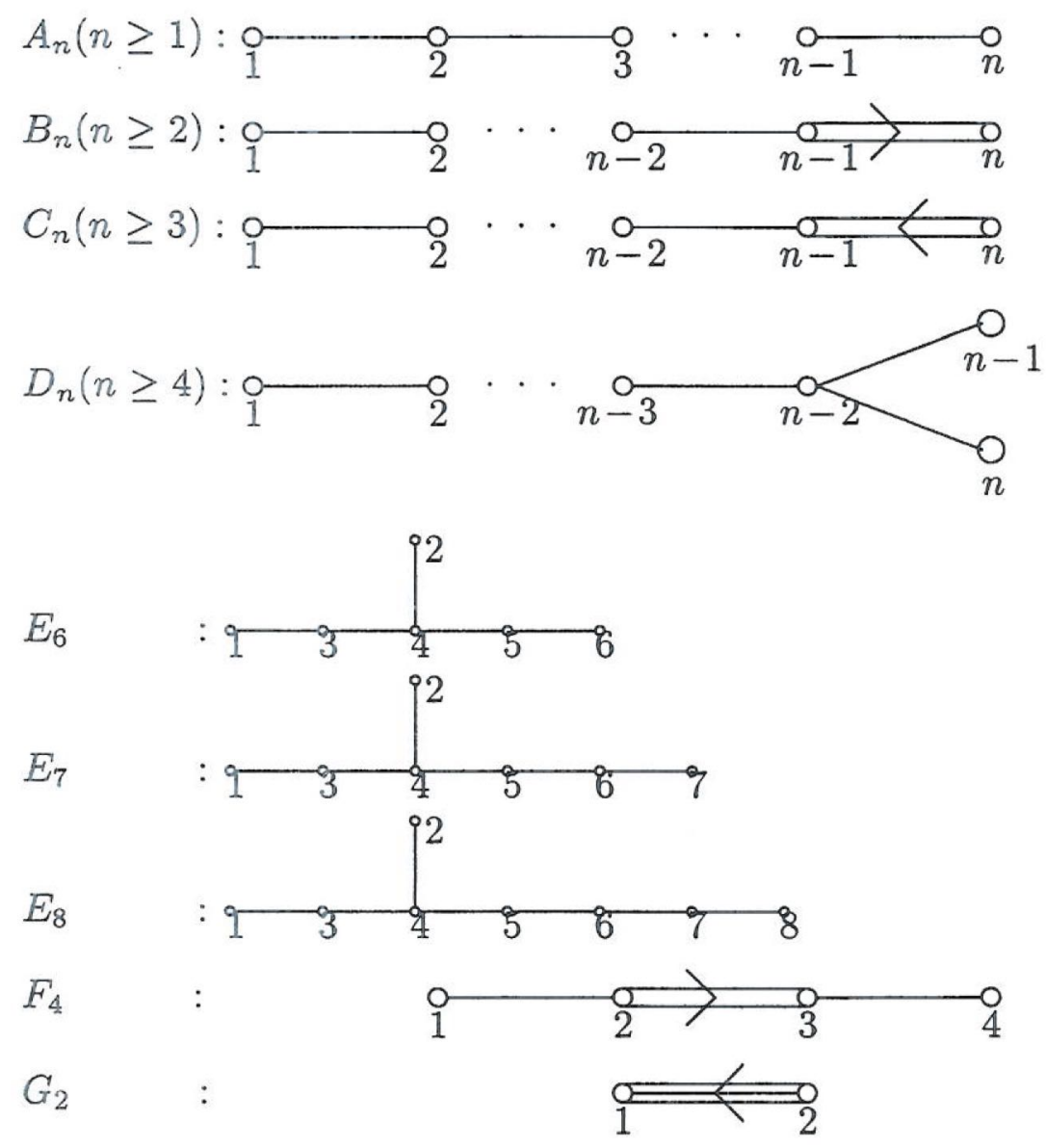

Fig. 1. Dynkin diagrams of irreducible root systems 
We have proved the Lemma for arbitrary subset $J$ of $I$. For the proof of the theorem, we need only those subsets of $J$ for which the corresponding root system $\Phi_{J}$ is irreducible i.e., its Dynkin diagram is connected. In fact, the choice of $J$ will be confined to those for which $\Phi_{J}$ is the root system of one of the following types: $A_{4}, B_{3}, C_{3}$ and $D_{4}$.

First we list the planar graphs $\Gamma(\Phi)$ where $\Phi$ is irreducible root system. $\Gamma\left(A_{1}\right)$ and $\Gamma\left(A_{2}\right)$ are totally disconnected graphs with 2 and 6 vertices respectively. $\Gamma\left(A_{3}\right)$ has 24 vertices and 8 disjoint edges. $\Gamma\left(B_{2}\right)$ has 8 vertices and 4 disjoint edges. $\Gamma\left(G_{2}\right)$ has 12 vertices and 12 edges as shown in Fig.2. All these are planar graphs. This leaves us with the following graphs:

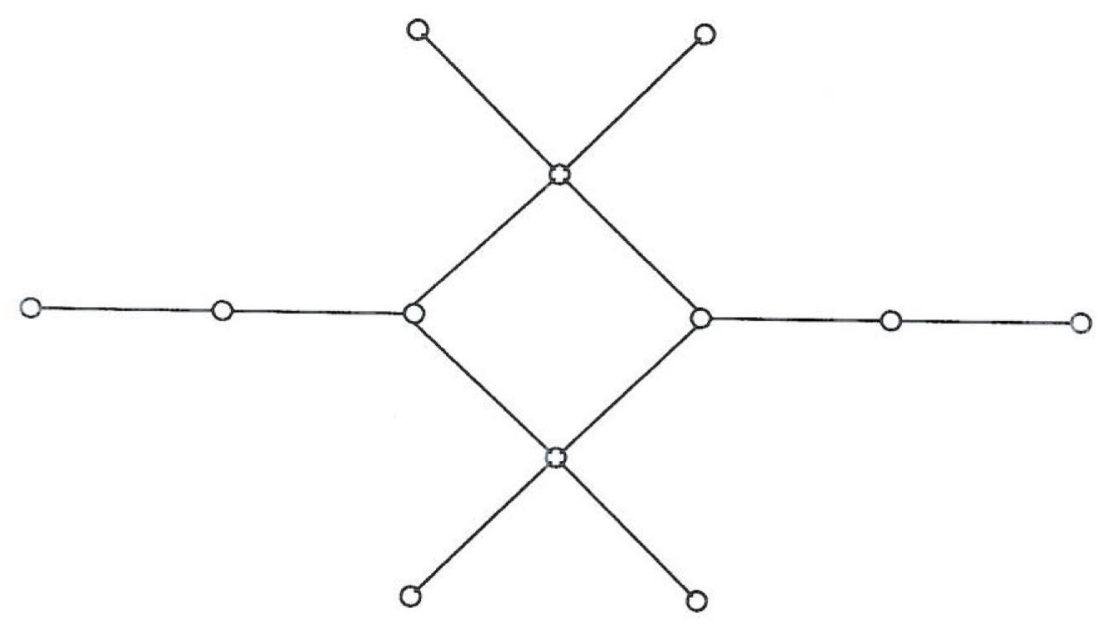

Fig. 2. The graph $\Gamma\left(G_{2}\right)$

$$
\left.\begin{array}{cccc}
\Gamma\left(A_{n}\right) \text { for } n \geq 4, & \Gamma\left(B_{n}\right) \text { for } n \geq 3, & \Gamma\left(C_{n}\right) \text { for } n \geq 3, & \Gamma\left(D_{n}\right) \text { for } n \geq 4, \\
\Gamma\left(E_{6}\right), & \Gamma\left(E_{7}\right), & \Gamma\left(E_{8}\right), & \Gamma\left(F_{4}\right) .
\end{array}\right]
$$

Our first goal is to show that $\Gamma(\Phi)$ given above in $\left(^{*}\right)$ are nonplanar. First we prove the following

Proposition. The graphs $\Gamma\left(A_{4}\right), \Gamma\left(B_{4}\right), \Gamma\left(C_{3}\right)$, and $\Gamma\left(D_{4}\right)$ are nonplanar.

Proof. Let $\sigma \in W$ have a reduced expression $R_{i_{1}} R_{i_{2}} \cdots R_{i_{k}}$ in terms of the generators $R_{i}, i=1,2, \ldots, n$. We write $\sigma$ as $i_{1} i_{2} \ldots i_{k}$. For example the element $R_{1} R_{2} R_{1} R_{3}$ is written as 1213. Let id be the identity element of $W$. We prove the proposition by exhibiting subgraph of $\Gamma(W)$ which is either contractible to the complete graph $K_{5}$ (as in the case of $\left.\Gamma\left(A_{4}\right)\right)$ or homeomorphic to the bigraph $K_{3,3}$ (as in the remaining cases). Then by the genralized form of the Kuratowski's theorem [2] it follows that $\Gamma(W)$ is nonplanar. We write the edge $(\sigma, \tau)$ in a subgraph of $\Gamma(W)$ with the convention that $\sigma \rightarrow \tau$, so that the verification of the claim will be easy to check from the data given in
the Tables. 
Table 1. $\epsilon_{\sigma}$ and $\epsilon_{\sigma \sigma_{0}}$ for some elements of $W\left(A_{4}\right)$.

\begin{tabular}{rlcc}
\hline S.No. & $\sigma$ & $\epsilon_{\sigma}$ & $\epsilon_{\sigma \sigma_{0}}$ \\
\hline 1. & $\sigma_{1}=i d$ & 0 & $-\lambda_{1}-\lambda_{2}-\lambda_{3}-\lambda_{4}$ \\
2. & $\sigma_{2}=4321$ & $-\lambda_{4}$ & $-\lambda_{1}-\lambda_{2}-\lambda_{3}+3 \lambda_{4}$ \\
3. & $\sigma_{3}=342312$ & $-\lambda_{3}$ & $-\lambda_{1}-\lambda_{2}+3 \lambda_{3}-\lambda_{4}$ \\
4. & $\sigma_{4}=234123$ & $-\lambda_{2}$ & $-\lambda_{1}+3 \lambda_{2}-\lambda_{3}-\lambda_{4}$ \\
5. & $\sigma_{5}=1234$ & $-\lambda_{1}$ & $3 \lambda_{1}-\lambda_{2}-\lambda_{3}-\lambda_{4}$ \\
6. & $\sigma_{6}=213$ & $\lambda_{1}-2 \lambda_{2}+\lambda_{3}+\lambda_{4}$ & $-\lambda_{1}+\lambda_{2}-\lambda_{3}-\lambda_{4}$ \\
7. & $\sigma_{7}=14232$ & $-2 \lambda_{1}+\lambda_{2}+\lambda_{3}-2 \lambda_{4}$ & $\lambda_{1}-\lambda_{2}-\lambda_{3}+\lambda_{4}$ \\
8. & $\sigma_{8}=324$ & $\lambda_{1}+\lambda_{2}-2 \lambda_{3}+\lambda_{4}$ & $-\lambda_{1}-\lambda_{2}+\lambda_{3}-\lambda_{4}$ \\
9. & $\sigma_{9}=2431214$ & $\lambda_{1}-2 \lambda_{2}+\lambda_{3}-2 \lambda_{4}$ & $-\lambda_{1}+\lambda_{2}-\lambda_{3}+\lambda_{4}$ \\
10. & $\sigma_{10}=3123431$ & $-2 \lambda_{1}+\lambda_{2}-2 \lambda_{3}+\lambda_{4}$ & $\lambda_{1}-\lambda_{2}+\lambda_{3}-\lambda_{4}$ \\
\hline
\end{tabular}

The graph $\Gamma\left(A_{4}\right)$ has 120 vertices and 180 edges. Consider the subgraph given by the 10 elements listed in Table 1. For $\lambda=x \lambda_{1}+y \lambda_{2}+z \lambda_{3}+t \lambda_{4} \in E$, the Weyl's dimension polynomial $D(\lambda)$ is $\Psi(x, y, z, t) / \Psi(1,1,1,1)$ where

$$
\Psi(x, y, z, t)=x y z t(x+y)(y+z)(z+t)(x+y+z)(y+z+t)(x+y+z+t) .
$$

The edges are $\left(\sigma_{1}, \sigma_{6}\right),\left(\sigma_{1}, \sigma_{7}\right),\left(\sigma_{1}, \sigma_{8}\right) ;\left(\sigma_{2}, \sigma_{7}\right),\left(\sigma_{2}, \sigma_{8}\right),\left(\sigma_{2}, \sigma_{9}\right) ;\left(\sigma_{3}, \sigma_{8}\right),\left(\sigma_{3}, \sigma_{9}\right)$, $\left(\sigma_{3}, \sigma_{10}\right) ;\left(\sigma_{4}, \sigma_{9}\right),\left(\sigma_{4}, \sigma_{10}\right),\left(\sigma_{4}, \sigma_{6}\right) ;\left(\sigma_{5}, \sigma_{10}\right),\left(\sigma_{5}, \sigma_{6}\right)$ and $\left(\sigma_{5}, \sigma_{7}\right)$. The subgraph is as shown in Fig.3. Identify the points $\sigma_{i}$ with $\sigma_{i+5}$ for $i=1,2, \ldots, 5$. From this it follows that the subgraph in Fig.3 is contractible to the complete graph $K_{5}$ and hence $\Gamma\left(A_{4}\right)$ is nonplanar.

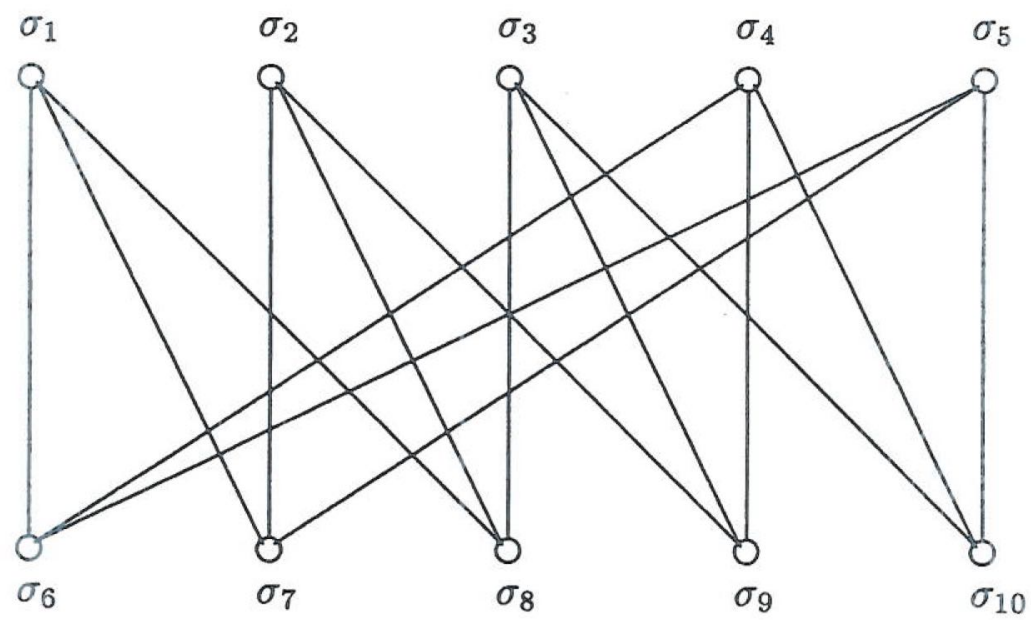

Fig. 3. A subgraph of $\Gamma\left(A_{4}\right)$ 
Table 2. $\epsilon_{\sigma}$ and $\epsilon_{\sigma \sigma_{0}}$ for some elements of $W\left(B_{3}\right)$.

\begin{tabular}{|c|c|c|c|}
\hline No. & $\sigma$ & $\epsilon_{\sigma}$ & $\epsilon_{\sigma \sigma_{0}}$ \\
\hline 1. & $\sigma_{1}=i d$ & 0 & $-\lambda_{1}-\lambda_{2}-\lambda_{3}$ \\
\hline 2. & $\sigma_{2}=23212$ & $2 \lambda_{1}-3 \lambda_{2}+2 \lambda_{3}$ & $-\lambda_{1}+\lambda_{2}-\lambda_{3}$ \\
\hline 3. & $\sigma_{3}=2312$ & $\lambda_{1}-2 \lambda_{2}+2 \lambda_{3}$ & $-\lambda_{1}+2 \lambda_{2}-3 \lambda_{3}$ \\
\hline 4. & $\sigma_{4}=1232$ & $-2 \lambda_{1}+\lambda_{2}$ & $2 \lambda_{1}-\lambda_{2}-\lambda_{3}$ \\
\hline 5. & $\sigma_{5}=123212$ & $-2 \lambda_{1}-\lambda_{2}+2 \lambda_{3}$ & $\lambda_{1}-\lambda_{3}$ \\
\hline 6. & $\sigma_{6}=212$ & $-\lambda_{1}-\lambda_{2}+4 \lambda_{3}$ & \\
\hline 7. & $\sigma_{7}=3212$ & $-\lambda_{1}+3 \lambda_{2}-4 \lambda_{3}$ & $-\lambda_{2}+\lambda_{3}$ \\
\hline 8. & $\sigma_{8}=12312$ & $-\lambda_{1}-\lambda_{2}+2 \lambda_{3}$ & $\lambda_{1}+\lambda_{2}-3 \lambda_{3}$ \\
\hline 9. & $\sigma_{9}=12$ & $-\lambda_{1}+2 \lambda_{2}$ & $\lambda_{1}-\lambda_{2}-\lambda_{3}$ \\
\hline 10. & $\sigma_{10}=12321$ & $-\lambda_{1}$ & $3 \lambda_{1}-\lambda_{2}-\lambda_{3}$ \\
\hline 12. & $\begin{array}{l}\sigma_{11}=132312 \\
\sigma_{12}=21323212\end{array}$ & $-\lambda_{1}+\lambda_{2}-2 \lambda_{3}$ & $\lambda_{1}-2 \lambda_{2}+3 \lambda_{3}$ \\
\hline 13. & $\sigma_{13}=312$ & $-\lambda_{1}+2 \lambda_{2}-2 \lambda_{3}$ & $\lambda_{1}-2 \lambda_{2}+\lambda_{3}$ \\
\hline 14. & $\sigma_{14}=1323212$ & $-2 \lambda_{1}+\lambda_{2}-2 \lambda_{3}$ & $\lambda_{1}-\lambda_{2}+\lambda_{3}$ \\
\hline
\end{tabular}

The graph $\Gamma\left(B_{3}\right)$ has 48 points and 100 edges. We list below in Table 2, the 14 elements of $W\left(B_{3}\right)$ which gives subgraph homeomorphic to the bigraph $\dot{K}_{3,3}$. For $\lambda=$ $x \lambda_{1}+y \lambda_{2}+z \lambda_{3} \in E$, the Weyl's dimension polynomial $D(\lambda)$ is $\Psi(x, y, z) / \Psi(1,1,1)$ where

$$
\Psi(x, y, z)=x y z(x+y)(y+z)(2 y+z)(x+y+z)(x+2 y+z)(2 x+2 y+z) .
$$

The edges are $\left(\sigma_{1}, \sigma_{2}\right),\left(\sigma_{1}, \sigma_{3}\right),\left(\sigma_{1}, \sigma_{4}\right),\left(\sigma_{3}, \sigma_{5}\right),\left(\sigma_{5}, \sigma_{6}\right),\left(\sigma_{4}, \sigma_{7}\right) ;\left(\sigma_{8}, \sigma_{2}\right),\left(\sigma_{8}, \sigma_{6}\right)$, $\left(\sigma_{8}, \sigma_{9}\right),\left(\sigma_{9}, \sigma_{7}\right) ;\left(\sigma_{10}, \sigma_{11}\right),\left(\sigma_{11}, \sigma_{12}\right),\left(\sigma_{12}, \sigma_{2}\right),\left(\sigma_{10}, \sigma_{13}\right),\left(\sigma_{13}, \sigma_{6}\right),\left(\sigma_{10}, \sigma_{14}\right)$, and $\left(\sigma_{14}, \sigma_{7}\right)$. The subgraph is shown in Fig.4. This shows that $\Gamma\left(B_{3}\right)$ is nonplanar, since it has a subgraph homeomorphic to the bigraph $K_{3,3}$.

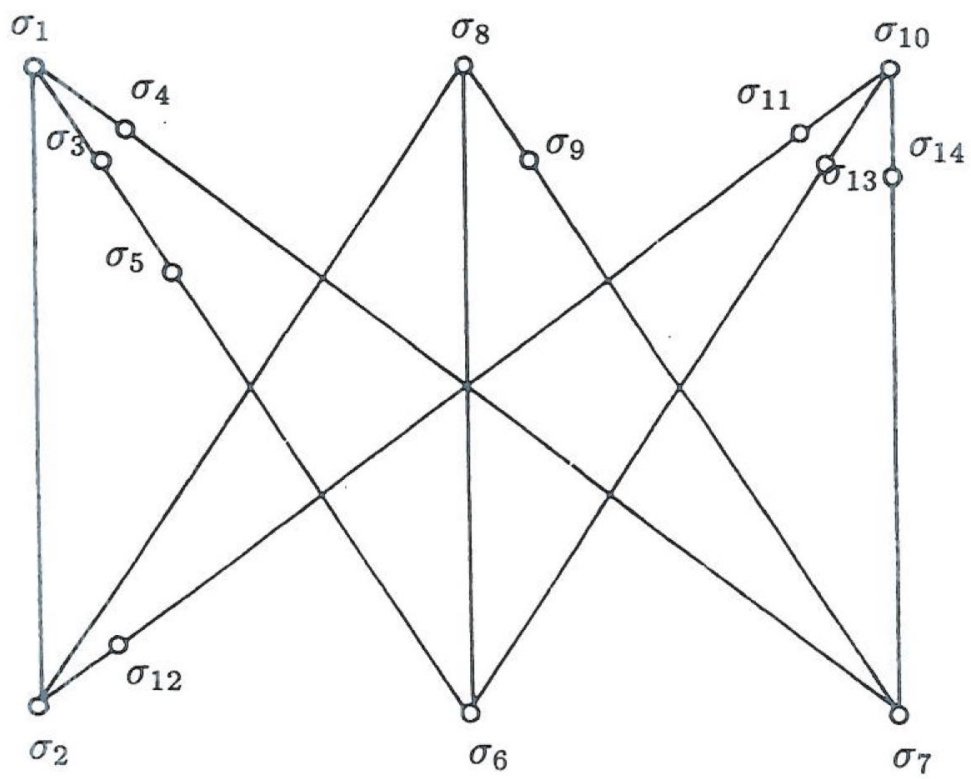

Fig. 4. A subgraph of $\Gamma\left(B_{3}\right)$

Table 3. $\epsilon_{\sigma}$ and $\epsilon_{\sigma \sigma_{0}}$ for some elements of $W\left(C_{3}\right)$. 


\begin{tabular}{rlcc} 
S.No. & $\sigma$ & $\epsilon_{\sigma}$ & $\epsilon_{\sigma \sigma_{0}}$ \\
\hline 1. & $\sigma_{1}=321$ & $-\lambda_{2}-\lambda_{3}$ & $-\lambda_{1}-3 \lambda_{2}+2 \lambda_{3}$ \\
2. & $\sigma_{2}=323121$ & $2 \lambda_{1}-\lambda_{2}-\lambda_{3}$ & $-2 \lambda_{1}+\lambda_{3}$ \\
3. & $\sigma_{3}=2323$ & $4 \lambda_{1}-\lambda_{2}-\lambda_{3}$ & $-\lambda_{1}$ \\
4. & $\sigma_{4}=3121$ & $-\lambda_{1}+3 \lambda_{2}-2 \lambda_{3}$ & $-2 \lambda_{2}+\lambda_{3}$ \\
5. & $\sigma_{5}=31213$ & $-2 \lambda_{1}+3 \lambda_{2}-2 \lambda_{3}$ & $\lambda_{1}-2 \lambda_{2}+\lambda_{3}$ \\
6. & $\sigma_{6}=323123121$ & $-\lambda_{1}-\lambda_{2}-\lambda_{3}$ & 0 \\
7. & $\sigma_{7}=3123121$ & $-2 \lambda_{1}+\lambda_{2}-\lambda_{3}$ & $2 \lambda_{1}-2 \lambda_{2}+\lambda_{3}$ \\
8. & $\sigma_{8}=23212323$ & $-\lambda_{2}-\lambda_{3}$ & $-\lambda_{1}+\lambda_{2}$ \\
9. & $\sigma_{9}=312132$ & $-\lambda_{1}+\lambda_{2}-\lambda_{3}$ & $2 \lambda_{1}-3 \lambda_{2}+2 \lambda_{3}$ \\
10. & $\sigma_{10}=3231213$ & $\lambda_{1}-\lambda_{2}-\lambda_{3}$ & $-\lambda_{1}+\lambda_{3}$ \\
11. & $\sigma_{11}=3213$ & $2 \lambda_{1}+\lambda_{2}-2 \lambda_{3}$ & $-\lambda_{1}-\lambda_{2}+\lambda_{3}$ \\
12. & $\sigma_{12}=321323$ & $-\lambda_{3}$ & $-\lambda_{1}-\lambda_{2}+2 \lambda_{3}$ \\
13. & $\sigma_{13}=323$ & $2 \lambda_{1}-\lambda_{3}$ & $-2 \lambda_{1}-\lambda_{2}+\lambda_{3}$ \\
14. & $\sigma_{14}=3$ & $2 \lambda_{2}-\lambda_{3}$ & $-\lambda_{1}-\lambda_{2}$ \\
15. & $\sigma_{15}=1323$ & $-2 \lambda_{1}+2 \lambda_{2}-\lambda_{3}$ & $2 \lambda_{1}-3 \lambda_{2}+\lambda_{3}$ \\
16. & $\sigma_{16}=3212323$ & $-\lambda_{1}+\lambda_{2}-2 \lambda_{3}$ & $-\lambda_{2}+\lambda_{3}$ \\
\hline
\end{tabular}

The graph $\Gamma\left(C_{3}\right)$ has 48 points and 96 edges. Consider the subgraph given by the 16 points listed below in the Table 3. For $\lambda=x \lambda_{1}+y \lambda_{2}+z \lambda_{3} \in E$, the Weyl's dimension polynomial $D(\lambda)$ is $\Psi(x, y, z) / \Psi(1,1,1)$ where

$$
\Psi(x, y, z)=x y z(x+y)(y+z)(y+2 z)(x+y+z)(x+y+2 z)(x+2 y+2 z) .
$$

The edges are $\left(\sigma_{1}, \sigma_{2}\right),\left(\sigma_{1}, \sigma_{3}\right),\left(\sigma_{4}, \sigma_{3}\right),\left(\sigma_{1}, \sigma_{5}\right),\left(\sigma_{5}, \sigma_{6}\right) ;\left(\sigma_{7}, \sigma_{8}\right) ;\left(\sigma_{2}, \sigma_{8}\right),\left(\sigma_{7}, \sigma_{4}\right)$, $\left(\sigma_{7}, \sigma_{6}\right) ;\left(\sigma_{9}, \sigma_{6}\right),\left(\sigma_{9}, \sigma_{4}\right),\left(\sigma_{9}, \sigma_{10}\right),\left(\sigma_{10}, \sigma_{11}\right),\left(\sigma_{12}, \sigma_{11}\right),\left(\sigma_{12}, \sigma_{13}\right),\left(\sigma_{13}, \sigma_{14}\right),\left(\sigma_{15}, \sigma_{14}\right)$, $\left(\sigma_{15}, \sigma_{16}\right)$, and $\left(\sigma_{2}, \sigma_{16}\right)$. The subgraph, which is homeomorphic to $K_{3,3}$, is shown in Fig.5. By generalized Kuratowski's theorem, $\Gamma\left(C_{3}\right)$ is nonplanar.

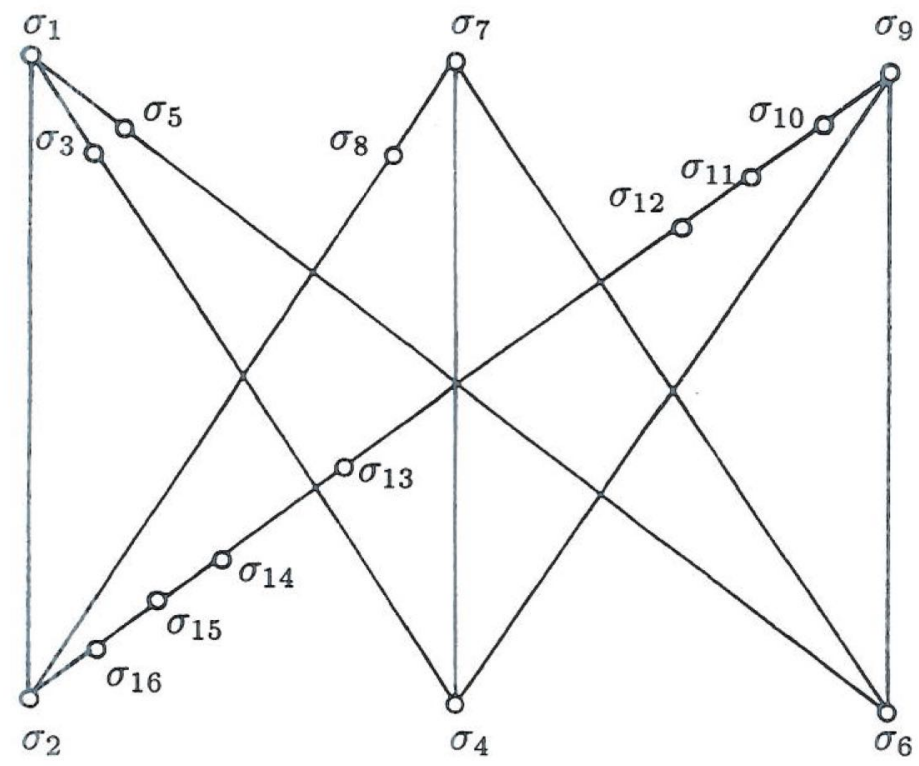

Fig. 5. A subgraph of $\Gamma\left(C_{3}\right)$ 
The graph $\Gamma\left(D_{4}\right)$ has 192 vertices and 624 edges. We list below in Table 4 the elements of $\Gamma\left(D_{4}\right)$ and consider its subgraph. For $\lambda=x \lambda_{1}+y \lambda_{2}+z \lambda_{3}+t \lambda_{4} \in E$, the Weyl's dimension polynomial $D(\lambda)$ is given by $\Psi(x, y, z, t) / \Psi(1,1,1,1)$ where

$$
\begin{aligned}
\Psi(x, y, z, t)= & x y z t(x+y)(y+z)(y+t)(x+y+z)(x+y+t)(z+y+t) \\
& \times(x+y+z+t)(x+2 y+z+t) .
\end{aligned}
$$

The edges in the subgraph are $\left(\sigma_{1}, \sigma_{7}\right),\left(\sigma_{1}, \sigma_{8}\right),\left(\sigma_{1}, \sigma_{9}\right) ;\left(\sigma_{2}, \sigma_{4}\right),\left(\sigma_{4}, \sigma_{7}\right),\left(\sigma_{2}, \sigma_{5}\right)$, $\left(\sigma_{5}, \sigma_{8}\right),\left(\sigma_{2}, \sigma_{6}\right),\left(\sigma_{6}, \sigma_{9}\right) ;\left(\sigma_{3}, \sigma_{7}\right),\left(\sigma_{3}, \sigma_{8}\right)$, and $\left(\sigma_{3}, \sigma_{9}\right)$. The subgraph, shown in Fig.6, is homeomorphic to $K_{3,3}$. Therefore $\Gamma\left(D_{4}\right)$ is nonplanar.

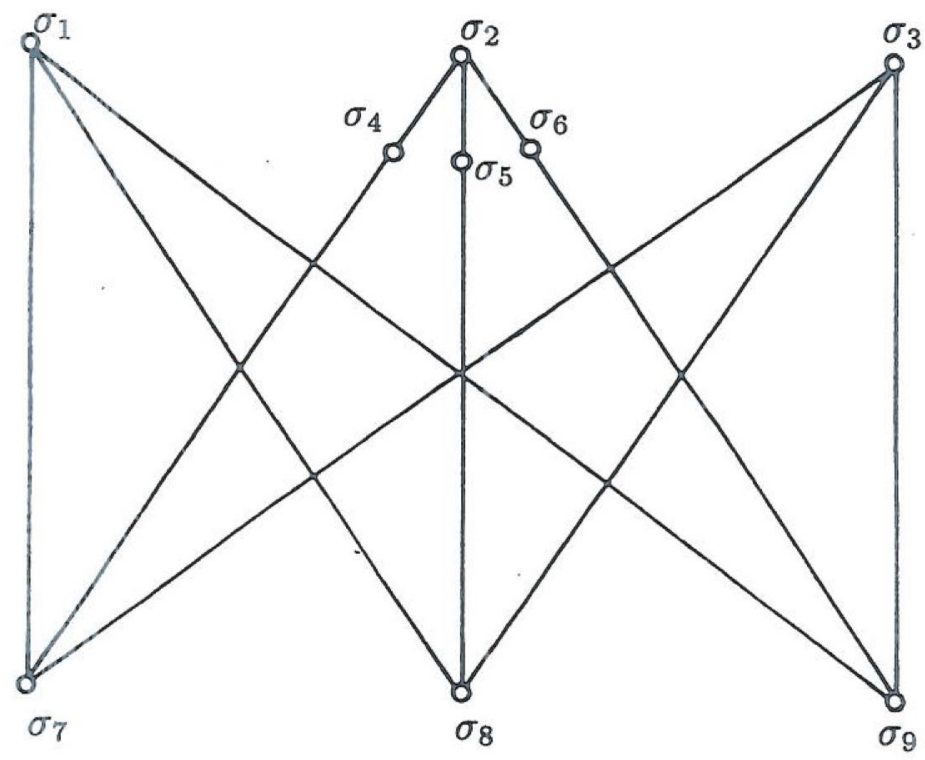

Fig. 6. A subgraph of $\Gamma\left(D_{4}\right)$

Table 4. $\epsilon_{\sigma}$ and $\epsilon_{\sigma \sigma_{0}}$ for some elements of $W\left(D_{4}\right)$.

\begin{tabular}{rlcc}
\hline S.No. & $\sigma$ & $\epsilon_{\sigma}$ & $\epsilon_{\sigma \sigma_{0}}$ \\
\hline 1. & $\sigma_{1}=2$ & $\lambda_{1}-\lambda_{2}+\lambda_{3}+\lambda_{4}$ & $-\lambda_{1}-\lambda_{2}-\lambda_{4}$ \\
2. & $\sigma_{2}=21342$ & $\lambda_{1}-2 \lambda_{2}+\lambda_{3}+\lambda_{4}$ & $-2 \lambda_{1}+3 \lambda_{2}-2 \lambda_{3}-2 \lambda_{4}$ \\
3. & $\sigma_{3}=213421342$ & $-\lambda_{2}$ & $-\lambda_{1}+3 \lambda_{2}-\lambda_{3}-\lambda_{4}$ \\
4. & $\sigma_{4}=121342134$ & $-\lambda_{1}-2 \lambda_{2}+\lambda_{3}+\lambda_{4}$ & $\lambda_{1}+\lambda_{2}-\lambda_{3}-\lambda_{4}$ \\
5. & $\sigma_{5}=321342134$ & $\lambda_{1}-2 \lambda_{2}-\lambda_{3}+\lambda_{4}$ & $-\lambda_{1}+\lambda_{2}+\lambda_{3}-\lambda_{4}$ \\
6. & $\sigma_{6}=421342134$ & $\lambda_{1}-2 \lambda_{2}+\lambda_{3}-\lambda_{4}$ & $-\lambda_{1}+\lambda_{2}-\lambda_{3}+\lambda_{4}$ \\
7. & $\sigma_{7}=12134$ & $-2 \lambda_{1}-\lambda_{2}+2 \lambda_{3}+2 \lambda_{4}$ & $\lambda_{1}-\lambda_{3}-\lambda_{4}$ \\
8. & $\sigma_{8}=32134$ & $2 \lambda_{1}-\lambda_{2}-2 \lambda_{3}+2 \lambda_{4}$ & $-\lambda_{1}+\lambda_{3}-\lambda_{4}$ \\
9. & $\sigma_{9}=42134$ & $2 \lambda_{1}-\lambda_{2}+2 \lambda_{3}-2 \lambda_{4}$ & $-\lambda_{1}-\lambda_{3}+\lambda_{4}$ \\
\hline
\end{tabular}

The proposition leads to the following 
Theorem. The graph $\Gamma(\Phi)$ is nonplanar when it is any one of the graphs in (*). In other words, for irreducible root system $\Phi, \Gamma(\Phi)$ is nonplanar except for $\Gamma\left(A_{1}\right), \Gamma\left(A_{2}\right), \Gamma\left(B_{2}\right)$ and $\Gamma\left(G_{2}\right)$.

Proof. The proof follows from the corollary of the lemma and the proposition. We indicate the choice of $J$ in each case with reference to Fig.1.

The graph $\Gamma\left(A_{4}\right)$ occurs as a subgraph in $\Gamma\left(A_{n}\right)$ for $n \geq 4$, as can be seen by choosing $J=\{1,2,3,4\}$ or any four indices of consecutive nodes in the Dynkin diagram of $A_{n}$ for $n \geq 4$. By the corollary of the lemma and the proposition it follows that $\Gamma\left(A_{n}\right)$ for $n \geq 4$ is nonplanar.

The graph $\Gamma\left(B_{3}\right)$ occurs as a subgraph in $\Gamma\left(B_{n}\right)$ for $n \geq 3$ with $J=\{n-2, n-1, n\}$ and in $\Gamma\left(F_{4}\right)$ with $J=\{1,2,3\}$. This implies that $\Gamma\left(B_{n}\right)$ for $n \geq 3$ and $\Gamma\left(F_{4}\right)$ are nonplanar.

The graph $\Gamma\left(C_{n}\right)$ for $n \geq 3$ is nonplanar since $\Gamma\left(C_{3}\right)$ occurs as a subgraph with $J=\{n-2, n-1, n\}$.

Finally, $\Gamma\left(D_{4}\right)$ occurs as a subgraph in $\Gamma\left(D_{n}\right)$ for $n \geq 4, \Gamma\left(E_{6}\right), \Gamma\left(E_{7}\right)$ and $\Gamma\left(E_{8}\right)$. This can be seen by choosing $J=\{n-3, n-2, n-1, n\}$ for the first case and $J=\{2,3,4,5\}$ for the remaining cases. Therefore, all of these graphs are nonplanar.

Remark. The graph $\Gamma\left(A_{4}\right)$ is nonplanar also implies that $\Gamma\left(B_{n}\right), \Gamma\left(C_{n}\right)$ and $\Gamma\left(D_{n}\right)$, all for $n \geq 5, \Gamma\left(E_{6}\right), \Gamma\left(E_{7}\right)$ and $\Gamma\left(E_{8}\right)$ are nonplanar. The choice of $J=\{1,2,3,4\}$ does it for the first two cases whereas for the remaining cases $J$ is any set of indices of 4 nodes which gives the Dynkin diagram of $A_{4} \cdot \Gamma\left(C_{3}\right)$ occurs as a subgraph of $\Gamma\left(F_{4}\right)$ with $J=\{2,3,4\}$ and this also shows that $\Gamma\left(F_{4}\right)$ is nonplanar.

\section{References}

[1] L. Chaskofsky, "Variation on Hulsurkar's matrix with applications to representation of algebraic Chevalley groups," J. Algebra, 82, 255-274, 1983.

[2] F. Harary, Graph Theory, Addison Wesley. Mass., 1972.

[3] S. G. Hulsurkar, "Proof of Verma's conjecture on Weyl's dimension polynomial," Inventiones Math., $27,45-52,1974$.

[4] J. E. Humphreys, Introduction to Lie Algebras and Representation Theory, Springer-Verlag, New York, 1972.

[5] Samy A. Youssef, Ph. D. Thesis, Indian Istitute of Technology, Kharagpur, July 1993. 